\title{
Tendenz zum präpositionalen Genitiv am Beispiel von entsprechend, gemäß und nahe
}

\author{
Radim MAŇÁK
}

\begin{abstract}
The tendency towards the prepositional genitive using the examples of entsprechend, gemäß and nahe

This article focuses on the use of the prepositional genitive instead of the dative for the prepositions entsprechend, gemäß and nahe. The aim of this study is to evaluate the frequency of genitives with these prepositions in current newspaper texts and to determine requirements for further research. The selected prepositions are described lexicographically, and the frequency of their genitive and dative usage is evaluated in a corpus developed by the author of this paper within DeReKo.
\end{abstract}

Keywords: prepositions, genitive, dative, language change

DOI: doi.org/10.15452/StudiaGermanistica.2021.28.0004

\section{Einleitung}

Der Wechsel des präpositionalen Kasus vom Dativ zum Genitiv hat in den letzten Jahrzehnten das wissenschaftliche Interesse erweckt. Jennifer Engemann (2013:118-119) untersuchte z. B. den Wechsel vom Dativ zum Genitiv in der Präpositionsrektion. Sie stellte fest, dass der präpositionale Genitiv nicht im Rückgang begriffen ist und nicht auf die Schriftsprache beschränkt ist. Weiter wurde bestätigt, ,dass die ,neue“ Genitivrektion standardsprachlich eher akzeptiert wird als die ,neue“ Dativrektion bei Genitivpräpositionen“. Als Gründe für den Wechsel nennt sie Grammatikalisierung, Sprachoptimierung, Prestigefaktoren und Unsicherheit im Sprachgebrauch. Di Meola erforschte die Genitivrektion der dativischen Präpositionen entgegen, nahe, entsprechend und gemä $\beta$ mit dem Schluss, dass „,selbst in kontrollierter Schriftsprache die Genitivrektion keine Seltenheit darstellt“ (Di Meola 1999:346). In einer weiteren Studie zu den semantisch irrelevanten Rektionsschwankungen konstatiert er, dass Rektionsschwankungen bei fast allen Typen der Präpositionen belegt sind und die Norminstanzen auf die Rektionsveränderungen mit einer gewissen Verzögerung reagieren. Interessant ist auch die Erkenntnis, dass ,die Tendenz zum Genitiv deutlich stärker als die Tendenz zum Dativ“ ist (Di Meola 2009:217-218).

Der Prozess der stufenartigen Wende vom Dativ zum Genitiv bei der Präposition entsprechend wird von Klein (2018:269) anschaulich dargelegt. 


\begin{tabular}{|l|l|l|}
\hline Das entspricht meinem Plan. & Dativ & Alt: syntaktische Konstruktion \\
\hline$\ldots$ meinem Plan entsprechend. & Dativ & syntaktische Konstruktion / Postposition (?) \\
\hline$\ldots$ entsprechend meinem Plan. & Dativ & Neu: Dativ-Präposition \\
\hline$\ldots$ entsprechend meines Plans. & Genitiv & Ganz neu: Genitiv-Präposition \\
\hline
\end{tabular}

Tab. 1: Entwicklung von entsprechend (Klein 2018:269)

Mein Artikel stellt eine Studie über die Tendenz des Wechsels vom präpositionalen Dativ zum präpositionalen Genitiv anhand dreier Präpositionen vor. Für die vorliegende Untersuchung wurden die Dativpräpositionen entsprechend, gemä $\beta$ und nahe gewählt, die auch in den zentralen Arbeiten zu dieser Frage behandelt werden, so dass ein Vergleich zwischen den dort gemachten Feststellungen möglich wird.

Die Duden-Grammatik (2016b:623) führt folgende Präpositionen an, die ursprünglich mit Dativ verbunden werden, bei denen es jedoch zur Genitivrektion kommt: entgegen, gemäß, laut, mitsamt, samt, entsprechend, nahe, dank. Di Meola (2014:139) ergänzt vor allem noch: inmitten, binnen, trotz, gegenüber, ähnlich, gleich. Den Anstoß für die Wahl gab die Literaturrecherche und die in den früheren Studien untersuchten Präpositionen. Sowohl in der Duden-Grammatik (2016b) als auch bei Di Meola (2014) werden die von mir ausgewählten Präpositionen behandelt. Auf der Basis der in diesem Beitrag vorgelegten Ergebnisse sollen auch weitere ursprüngliche Dativpräpositionen untersucht werden, die zur Verwendung mit Genitiv neigen.

Das Ziel der vorliegenden Studie ist, die Häufigkeit der genitivischen Rektion bei den ausgewählten Präpositionen in aktuellen Zeitungstexten auszuwerten und damit gleichzeitig die Voraussetzungen für die weitere Forschung zu legen.

\section{Korpus und Methode der Untersuchung}

Die Untersuchung erfolgt in folgenden Phasen.

Die erste Phase besteht in der lexikographischen Beschreibung der gewählten Präpositionen. Die Rektion der Präpositionen wird in ,Duden-Online', ,Wahrig. Wörterbuch der deutschen Sprache ' und in den Wörterbüchern der Zweifelsfälle, ${ }^{1}$ namentlich in ,Duden. Das Wörterbuch der sprachlichen Zweifelsfälle' aus dem Jahr 2016, und in ,Wahrig. Richtiges Deutsch leicht gemacht‘ aus dem Jahr 2009, überprüft. Dieses Vorgehen reflektiert den Tatbestand, dass die Erforschung von Standardsprachlichkeit ,nicht nur eine usus-orientierte, objektsprachliche Dimension, sondern auch eine kodex-orientierte, metasprachliche Komponente" (Klein 2013:29) hat.

In der zweiten Phase wird eine korpusbasierte Untersuchung durchgeführt, die den aktuellen Gebrauch dokumentiert. Mit diesem Vorgehen wird Kleins (2013:27) Feststellung entsprochen, „dass die Frage danach, was gegenwärtig als standardsprachlich bzw. nicht-standardsprachlich zu gelten hat, vernünftigerweise nur usus-orientiert zu beantworten ist.“

Das Korpus wurde aus den Ausgaben von Zeitungstexten (vor allem Tageszeitungen) vom August 2019 zusammengestellt. In der folgenden Tabelle 2 sind die Zeitungen aufgelistet, die das Korpus der vorliegenden Untersuchung ausmachen. Bei der Zuordnung der Region, aus der die jeweilige Zeitung stammt, wurde auf die im Variantenwörterbuch (Ammon/Bickel/ Lenz 2016:LIII) präsentierte Karte zurückgegriffen.

\begin{tabular}{|l|l|l|}
\hline Zeitung & Sitz & Region \\
\hline Berliner Morgenpost & Berlin & Deutschland-Nord \\
\hline Berliner Zeitung & Berlin & Deutschland-Nord \\
\hline die tageszeitung & Berlin & Deutschland-Nord \\
\hline
\end{tabular}

Die Bedeutung dieser Wörterbücher für die Untersuchung kann dadurch akzentuiert werden, dass Klein (2014:234)

Zweifelsfälle-Wörterbücher von Duden und Wahrig zum Kernkodex der Sprache ordnet. 


\begin{tabular}{|l|l|l|}
\hline Hamburger Morgenpost & Hamburg & Deutschland-Nord \\
\hline Hannoversche Allgemeine & Hannover & Deutschland-Nord \\
\hline Nordkurier & Neubrandenburg & Deutschland-Nord \\
\hline Rhein-Zeitung & Koblenz & Deutschland-Mitte \\
\hline Süddeutsche Zeitung & München & Deutschland-Süd \\
\hline Mannheimer Morgen & Mannheim & Deutschland-Süd \\
\hline Nürnberger Nachrichten & Nürnberg & Deutschland-Süd \\
\hline Nürnberger Zeitung & Nürnberg & Deutschland-Süd \\
\hline Die Presse & Wien & Österreich \\
\hline Niederösterreichische Nachrichten & St. Pölten & Österreich \\
\hline Neue Zürcher Zeitung & Zürich & Schweiz \\
\hline St. Galler Tagblatt & St. Gallen & Schweiz \\
\hline Tages-Anzeiger & Zürich & Schweiz \\
\hline
\end{tabular}

Tab. 2: Aufbau des Korpus (Autor dieses Artikels)

Ziel dieser Untersuchung ist es, die Rektion der ausgewählten Präpositionen in standardsprachlichen Texten zu ermitteln. Der redaktionelle Teil überregionaler Zeitungen gilt als typischer Ort von Texten, die einer standardsprachlichen Norm folgen: „Standardsprachlichkeit manifestiert sich demgemäß durch die kommunikative Funktion zur Konstruktion des höchsten Grades an Öffentlichkeit, wie es durch überregionale Pressetexte geschieht" (Scherr/Niehaus 2013:76). ${ }^{2}$ Die Texte aus den überregionalen Zeitungen wurden in dem verwendeten Korpus noch um Texte aus Regionalzeitungen ergänzt.

Die korpuslinguistische Analyse des präpositionalen Kasus wurde in folgenden Schritten durchgeführt:

1. Korpussuche.

Die Zahl der Treffer war in manchen Fällen groß. Um die manuelle Bearbeitung der Treffer zu ermöglichen, wurde nur eine begrenzte Zahl von ihnen bearbeitet. Wenn die Zahl der Treffer 1000 nicht überschritt, wurden alle gefundenen Treffer bewertet. Wenn dagegen die Zahl der Treffer 1000 überschritt, wurden die Treffer in Cosmas zufällig sortiert und die ersten 1000 Treffer analysiert. Diese Vorgehensweise gewährleistet, dass in der Auswahlmenge von 1000 Treffern eine ähnliche Verteilung des gesuchten Phänomens wie in der ganzen Treffermenge vorliegt.

2. Manuelle Aussortierung der Treffer.

Die Treffer mussten manuell aussortiert werden, um falsche Treffer zu vermeiden. Es geht dabei vor allem um Feminina, bei denen nicht entschieden werden kann, ob es sich um den Dativ oder den Genitiv handelt.

3. Erstellung einer Excel-Datenbank.

Es wurde eine Excel-Datenbank der Treffer gebildet, wobei den Treffern klassifizierende Zeichen zugeordnet wurden. Diese Zeichen ermöglichen eine effiziente Sortierung und Analyse der Treffer.

\section{Ergebnisse}

Die Präposition entsprechend wird von Duden-Online (C2020) und Wahrig (2009:468) als Präposition mit Dativ eingestuft. Duden (2016a:288) lässt mehr Variation zu, indem dieses Nachschlagewerk dieser Präposition meist den Dativ zuweist; der Genitiv sei ein Zeichen der Hyperkorrektheit. ${ }^{3}$

2 Auch für die vorgesehenen diachronen Untersuchungen wäre die Zeitungssprache seit dem 18. Jh. ein geeignetes Objekt (Niehaus 2016:19).

3 In Wahrig (2018:306) wird die Form entsprechend als Partizip Präsens von entsprechen und als Adjektiv betrachtet, eine explizite Angabe als Präposition fehlt. 
Die empirischen Ergebnisse sind weniger eindeutig als die lexikographische Beschreibung, es findet sich ein bemerkenswerter Anteil genitivischer Verwendungen. Die dativische Rektion überwiegt mit 83 Fällen, in 25 Fällen steht der Genitiv.

Der Genitiv erscheint im Singular:

Saubere Einweggläser oder kleine Glasflaschen mit Schraubdeckel, wiederverwendbare Metallstrohhalme, Ösen (am besten im Durchmesser des Strohhalmes) mit entsprechendem Ösenwerkzeug, Hammer, Metallbohrer (entsprechend des Ösendurchmessers) und feines Schleifpapier. (Nordkurier 30.8.2019:22)

Unbestritten dürfte sein, dass ein zentrales Krankenhaus mit rund 320 Betten entsprechend des neuen Krankenhausplanes wirtschaftlicher arbeiten kann, als ein Krankenhaus mit zwei Standorten in einer Entfernung von 15 Kilometern. (Rhein-Zeitung 17.8.2019:20)

Etwas häufiger findet sich die genitivische Rektion im Plural:

Die Bahn müsse Gepäckstücke der Umsteiger entsprechend der hohen Sicherheitsanforderungen des Luftverkehrs aufnehmen und zuverlässig an die Airlines übergeben, verlangte BDL-Hauptgeschäftsführer Matthias von Randow während einer Onlinepressekonferenz. (Berliner Zeitung 17.8.2019:7)

Die Kreuzschifffahrt gilt unter anderem wegen des Einsatzes von Schweröl und entsprechend hoher Emissionswerte als umweltschädlich. (Berliner Zeitung 13.8.2019:7)

Mit einem Anteil von fast einem Viertel genitivischer Belege weicht der Befund der Korpusanalyse von der Regelung der Wörterbücher ab. Meiner Meinung nach kann eine so große Vertretung des Genitivs nicht als vereinzelter Fehler erklärt werden, sondern sie zeigt auf einen Wechsel vom Dativ zum Genitiv, der von den Wörterbüchern aufgrund der Korpusbefunde nicht berücksichtigt werden konnte.

Die Präposition gemäß regiert laut Duden-Online (C2020), Wahrig (2018:406), Wahrig (2009:465) und Duden (2016a:359) den Dativ. Die genitivische Verwendung wird von Wahrig (2009) als standardsprachlich nicht korrekt und von Duden (2016a:359) als Zeichen der Hyperkorrektheit eingestuft.

Nach der Aussortierung der unerwünschten Treffer (vor allem Feminina, bei denen Genitiv und Dativ zusammenfallen), sehen die Ergebnisse wie folgt aus. Hier überwiegt im Zeitungskorpus die Dativ-Rektion mit 304 Fällen ganz eindeutig; nur in 29 Fällen tritt ein Genitiv auf. Weitere 191 Treffer könnten aufgrund ihrer fehlenden Markierung im Singular sowohl den Genitiv wie den Dativ indizieren, wegen dieser Ambiguität werden sie in den weiteren Vergleich nicht einbezogen. ${ }^{4}$

Als Beispiele der Genitiv-Rektion im Singular (insgesamt 9 Fälle) können folgende Sätze angeführt werden:

Von den militärischen Gebäuden der Nazis ist nichts mehr zu sehen, sie wurden gemäß des Potsdamer Abkommens allesamt gesprengt. (Süddeutsche Zeitung 29.8.2019:35)

Gemäss des Zeitungsberichts im „Toggenburger Tagblatt“ sollte im Mitteilungsblatt der Gemeinde Kirchberg bezüglich Hochwasserschutzprojekt in Gähwil Klarheit in Bezug auf das weitere Vorgehen geschaffen werden. (St. Galler Tagblatt 19.8.2019)

Auch bei dieser Präposition ist die Genitivverwendung im Plural etwas häufiger (20 Fälle), und zwar mit bestimmtem Artikel oder Pronomen vor dem Substantiv, oder auch in Konstruktionen ohne Artikel:

Doch mit der Registrierung, der Kontrolle und der Vergabe des Kredits ist der Prozess nicht abgeschlossen: Die Daten müssen gemäß der Geldwäsche-Verordnungen laufend überprüft und aktuali-

$4 \quad$ Es ist hier auch von Bedeutung, dass bei artikellosem Gebrauch der regierten Substantive keine Kasusmarkierung eintritt. 
siert werden. (Die Presse 23.8.2019:17)

Gemäß dieser Umfragen wären in beiden Bundesländern nach der Wahl am 1. September nur Koalitionen mit mindestens drei Parteien möglich. (Berliner Morgenpost 28.8.2019:3)

Man erwartete, dass das Land Rheinland-Pfalz, gemäß gesetzlicher Vorgaben, wie bei anderen Privatschulen auch einen Teil des Kaufpreises übernehmen würde. (Rhein-Zeitung 13.8.2019:13)

Seit 2009 war zusammen mit Denkmalschützern der Originalzustand des barocken Parks gemäß alter Pläne wieder hergestellt worden. (Nordkurier 15.8.2019:4)

Im Vergleich mit dem Befund bei der Präposition entsprechend ist der Anteil der genitivischen Rektion bei gemäß ziemlich niedrig, er liegt bei $9 \%$. Es ist fraglich, ob bei solch einem geringen Wert von einem Wechsel gesprochen werden sollte. Angesichts des dezidiert standardsprachlichen Charakters des verwendeten Korpus und des Prestige-Niveaus der Zeitungstexte nehme ich an, dass auch dieses Ergebnis als Indiz für den Wechsel vom Dativ zum Genitiv gewertet werden kann.

Die Präposition nahe wird von Duden-Online (@2020), Wahrig (2018:679), Wahrig (2009:468) und auch Duden (2016a:651) einstimmig als Präposition mit Dativ eingestuft.

Die Korpusdaten aus dem Zeitungskorpus zeigen jedoch keine so eindeutigen Ergebnisse. Die Verwendung mit dem Dativ wurde in 83 Fällen festgestellt, die Verwendung mit dem Genitiv in 46 Fällen. Eine neutrale Form im Singular, die sich als Nominativ, Dativ und Akkusativ bzw. als Genitiv mit einer fehlenden s-Markierung interpretieren lässt, wurde in 116 Fällen identifiziert. Diese Formen wurden in den weiteren Genitiv/Dativ-Vergleich nicht einbezogen.

Die Rektionsvariation zwischen dem Genitiv und Dativ kann an den folgenden zwei Beispielen dokumentiert werden:

Auch der Ausländerbeauftragte Gerd Mackenroth hat Maaßen eingeladen, nach Riesa, in einen Hotelsaal nahe dem Bahnhof. (Hannoversche Allgemeine 19.8.2019:2)

Auch der Landes-Ausländerbeauftragte Gerd Mackenroth hat Maaßen eingeladen, nach Riesa in einen Hotelsaal nahe des Bahnhofs. (Berliner Zeitung 20.8.2019:3)

Es geht um eine weitgehend identische Formulierung, die in zwei verschiedenen Zeitungen publiziert und unterschiedlich korrigiert wurde. Die Verwendung verschiedener Kasus im gleichen Satzkontext zeugt von einer Kasus-Variation ohne semantische oder kontextuelle Differenzierung.

Es wurden 39 Belege der genitivischen Rektion im Singular identifiziert:

Der Fundort der Leichen liegt etwa acht Kilometer entfernt von der Stelle nahe des Städtchens Gillam, an der vor zweieinhalb Wochen das Fluchtauto der Teenager gefunden worden war. (Süddeutsche Zeitung 9.8.2019:10)

Danach sollten mit dem Bahnhofsausbau jeweils beide Endpunkte der Unterführung - einmal in der Teterower Straße und einmal nahe des Bahnhofsgebäudes - mit Fahrstühlen versehen werden. (Nordkurier 23.8.2019:15)

Er verfügt über einen Garten, liegt nahe eines Spielplatzes und ist verkehrstechnisch gut erreichbar. (St. Galler Taglbatt 16.8.2019)

Nicht so in der kargen und bis heute eher unberührten Region nahe eines Kaltzeitgletschers. (Süddeutsche Zeitung 12.8.2019:14)

Wenn auch in geringerem Ausmaß, finden sich doch auch 7 Fälle für den Genitiv im Plural:

Mitte Mai war nahe der Pyramiden von Giseh zudem ein Sprengsatz explodiert, als ein Bus mit Touristen vorbeifuhr. (Nordkurier 6.8.2019:8)

Deshalb wurden von 2011 an sogar lokale Oppositionsgruppen unterstützt, die nahe der von Israel annektierten Golanhöhen gegen Assads Soldaten kämpften, sie bekamen Geld und leichte 
Waffen, wie Israels ehemaliger Generalstabschef Gadi Eisenkot offenbarte. (Süddeutsche Zeitung 3.8.2019:13)

Die Diskrepanz zwischen der Regelung in den Wörterbüchern und den empirischen Ergebnissen ist im Fall der Präposition nahe am deutlichsten. Mehr als ein Drittel der Belege mit eindeutiger Rektion kann dem Genitiv zugeordnet werden, was die Annahme eines Rektionswechsels bestätigen lässt.

\section{Zusammenfassung und Diskussion}

Die zur lexikografischen Beschreibung verwendeten Wörterbücher stehen in relativem Einklang, was die Einstufung der untersuchten Präpositionen zum Kasus betrifft. Die Korpusanalyse bringt jedoch ein anderes Bild über den Usus in den deutschsprachigen Zeitungstexten, wo die Genitivrektion dieser Präpositionen relativ etabliert ist.

Die quantitative Analyse zeigt, dass die Präposition nahe den höchsten Anteil der genitivischen Rektion aufweist, was die Daten von Duden (2016b) bestätigt, jedoch im Kontrast zu Di Meola (2014) steht, der der Präposition gemäß die höchsten Anteile zuweist. Im Einklang mit Duden (2016b) wurde festgestellt, dass die genitivische Rektion öfter im Plural als im Singular erscheint.

\begin{tabular}{|l|l|l|l|}
\hline Präposition & Genitiv-Anteil & $\begin{array}{l}\text { Genitiv-Anteil nach } \\
\text { Duden-Grammatik } \\
(\mathbf{2 0 1 6 b : 6 2 3})\end{array}$ & $\begin{array}{l}\text { Genitiv-Anteil nach Di } \\
\text { Meola (2014:209) }\end{array}$ \\
\hline entsprechend & $\begin{array}{l}23,1 \%(\mathrm{n}=25) \\
\text { Sg. } 20,5 \%(\mathrm{n}=9) \\
\text { Pl. } 25,0 \%(\mathrm{n}=16)\end{array}$ & $\begin{array}{l}\text { Sg. } 12 \% \\
\text { Pl. } 28 \%\end{array}$ & $11 \%$ \\
\hline gemäß & $\begin{array}{l}8,7 \%(\mathrm{n}=29) \\
\text { Sg. } 5,0 \%(\mathrm{n}=9) \\
\mathrm{Pl} .13,2 \%(\mathrm{n}=20)\end{array}$ & $\begin{array}{l}\text { Sg. } 14 \% \\
\text { Pl. } 22 \%\end{array}$ & $25 \%$ \\
\hline nahe & $\begin{array}{l}35,7 \%(\mathrm{n}=46) \\
\text { Sg. } 33,3 \%(\mathrm{n}=39) \\
\mathrm{Pl} .58,3 \%(\mathrm{n}=7)\end{array}$ & $\begin{array}{l}\text { Sg. } 31 \% \\
\text { Pl. } 81 \%\end{array}$ & $8 \%$ \\
\hline
\end{tabular}

Tab. 3: Genitiv-Anteil der Dativ-Präpositionen (eigene Forschung, DeReKo)

Wenn der Aspekt der Arealität betrachtet wird, zeigt sich, dass die genitivische Rektion öfter im Norden des deutschsprachigen Gebiets als im Süden erscheint. Bei der Präposition nahe ist diese Tendenz jedoch schwächer ausgeprägt, weil sie in der Region Deutschland-Nord schwächer vertreten ist als in den Regionen Deutschland-Mitte und Deutschland-Süd.

\begin{tabular}{|l|l|l|l|l|l|}
\hline Präposition & $\begin{array}{l}\text { Genitiv-Anteil } \\
\text { in D-Nord }\end{array}$ & $\begin{array}{l}\text { Genitiv-Anteil } \\
\text { in D-Mitte }\end{array}$ & $\begin{array}{l}\text { Genitiv-Anteil } \\
\text { in D-Süd }\end{array}$ & $\begin{array}{l}\text { Genitiv-Anteil } \\
\text { in AU }\end{array}$ & $\begin{array}{l}\text { Genitiv-Anteil } \\
\text { in CH }\end{array}$ \\
\hline entsprechend & $\begin{array}{l}29,6 \% \\
(\mathrm{n}=8)\end{array}$ & $\begin{array}{l}27,3 \% \\
(\mathrm{n}=9)\end{array}$ & $\begin{array}{l}22,2 \% \\
(\mathrm{n}=4)\end{array}$ & $\begin{array}{l}16,7 \% \\
(\mathrm{n}=2)\end{array}$ & $\begin{array}{l}11,1 \% \\
(\mathrm{n}=2)\end{array}$ \\
\hline gemäß & $\begin{array}{l}46,7 \% \\
(\mathrm{n}=7)\end{array}$ & $\begin{array}{l}38,7 \% \\
(\mathrm{n}=12)\end{array}$ & $\begin{array}{l}13,0 \% \\
(\mathrm{n}=3)\end{array}$ & $\begin{array}{l}16,7 \% \\
(\mathrm{n}=3)\end{array}$ & $\begin{array}{l}1,6 \% \\
(\mathrm{n}=4)\end{array}$ \\
\hline nahe & $\begin{array}{l}30,4 \% \\
(\mathrm{n}=17)\end{array}$ & $\begin{array}{l}48,3 \% \\
(\mathrm{n}=14)\end{array}$ & $\begin{array}{l}60,0 \% \\
(\mathrm{n}=9)\end{array}$ & $\begin{array}{l}26,7 \% \\
(\mathrm{n}=4)\end{array}$ & $\begin{array}{l}14,3 \% \\
(\mathrm{n}=2)\end{array}$ \\
\hline
\end{tabular}

Tab. 4: Genitiv-Anteil der Dativ-Präpositionen nach der Region (eigene Forschung, DeReKo)

Die identifizierten Zeichen der Unsicherheit in der Verwendung des Kasus auch bei geschulten Schreibern deuten darauf hin, dass es sich bei den untersuchten Fällen der präpositionalen Rektion um Zweifelsfälle handelt, die Anzeichen von Sprachwandel sein können. Bei der Suche nach der Erklärung dieses Phänomens kann der Charakter des verwendeten Korpus berücksichtigt werden. Die Zeitungstexte können als Modelltexte betrachtet werden, die mit einem bestimmten Prestige 
verbunden werden. In diesem Kontext ist das höhere Prestige von Bedeutung, das der Genitiv als Präpositionalkasus besitzt. Das könnte der Grund für die Akzeptanz der genitivischen Rektion in den Zeitungstexten sein, auch wenn diese Rektion der Regelung in den Wörterbüchern nicht entspricht.

Es wurde weiter festgestellt, dass die Wörterbücher bei der Reflexion der realen Kasusverwendung in Verzug geraten sind und nicht den aktuellen Stand der Verwendung widerspiegeln.

Die hier gewählte methodische Vorgehensweise erwies sich als anwendbar für die weitere geplante Forschung. Die manuelle Aussortierung und Bearbeitung der Treffer stellen zwar eine zeitliche Herausforderung dar und sind anfällig für Fehler in Hinblick auf den Human Factor, ermöglichen jedoch die Aufnahme unterschiedlicher Satzkonstruktionen mit Präposition (z. B. mit dem Adjektiv und ohne Artikel vor dem Substantiv) in die Korpusstudie.

Zudem kann auch diese kleine Studie schon etwas zur terminologischen Klärung beitragen. In der bisherigen Fachliteratur wird nur selten zwischen Wechsel und Schwankung unterschieden. Auch wenn ich bei meiner Korpusanalyse einige Male auf einen möglichen Rektionswechsel hingewiesen habe, ist das erste Ergebnis einer solchen Korpusarbeit wohl nur die Sichtbarkeit einer Schwankung zwischen zwei Möglichkeiten.

Demgegenüber wäre ein Wechsel ein sprachgeschichtlicher Vorgang, bei dem ein regierter Kasus durch einen anderen abgelöst wird. Um einen solchen Wechsel festzustellen, wäre ein diachron organisiertes Korpus notwendig. ${ }^{5}$

\section{Literaturverzeichnis}

\section{Primärquellen:}

Das Deutsche Referenzkorpus DeReKo. Mannheim. Zugänglich unter: https://cosmas2.ids-mannheim.de/cosmas2-web/ [28.8.2020].

\section{Sekundärliteratur:}

Ammon, Ulrich / Bickel, Hans / Lenz, Alexandra N. (Hrsg.) (2016): Variantenwörterbuch des Deutschen. Die Standardsprache in Österreich, der Schweiz, Deutschland, Liechtenstein, Luxemburg, Ostbelgien und Südtirol sowie Rumänien, Namibia und Mennonitensiedlungen. Berlin.

Di Meola, Claudio (1999): Entgegen, nahe, entsprechend und gemäß. Dativpräpositionen mit Genitivrektion. In: Zeitschrift für germanistische Linguistik, Nr. 3, S. 344-351. Zugänglich unter: https://www.degruyter.com/view/journals/zfgl/27/3/article-p309.xml [28. 8. 2020].

Di Meola, Claudio (2009): Rektionsschwankungen bei Präpositionen - erlaubt, verboten, unbeachtet. In: Konopka, Marek / Strecker, Bruno: Deutsche Grammatik-Regeln, Normen, Sprachgebrauch. Berlin, S. 195-221.

Di Meola, Claudio (2014): Die Grammatikalisierung deutscher Präpositionen. Tübingen.

Duden (2016a): Das Wörterbuch der sprachlichen Zweifelsfälle: Richtiges und gutes Deutsch. Berlin.

Duden (2016.): Die Grammatik: Unentbehrlich für richtiges Deutsch. Berlin.

Duden (@2020): Duden-Online. Zugänglich unter: https://www.duden.de/ [28.8.2020].

Eichinger, Ludwig M. / Rothe, Astrid (2014): Der Fall der Fälle. Entwicklungen in der nominalen Morphologie In: Plewnia, Albrecht / WitT, Andreas (Hrsg.): Sprachverfall? Dynamik - Wandel - Variation (= Institut für Deutsche Sprache Jahrbuch 2013). Berlin; Boston, S. 71-97.

EngEMANN, Jennifer (2013): Veränderungen in der Präpositionsrektion: Der Wechsel vom Dativ (bzw. Akkusativ) zum Genitiv. In: Triangulum. Germanistisches Jahrbuch 2012 für Estland, Lettland und Litauen, S. 97-125. Zugänglich unter: http://www.daad.lv/images/Triangulum/ triangulum_2012.pdf [16.8.2020]

KLEIN, Wolf Peter (2013): Warum brauchen wir einen klaren Begriff von Standardsprachlichkeit und wie könnte er gefasst werden? In: Hagemann, Jörg / Klein, Wolf Peter / Staffeldt, Sven (Hrsg.): Pragmatischer Standard. Tübingen, S. 15-33.

\footnotetext{
Vgl. Eichinger/Rothe (2014): eine Diachronie über das 20. Jahrhundert hin sowie das ganze im Artikel zitierte Akade-
} mie-Projekt, in dem Eichinger (2013) die Flexion behandelt. 
KLeIN, Wolf Peter (2014): Gibt es einen Kodex für die Grammatik des Neuhochdeutschen und, wenn ja, wie viele? Oder: Ein Plädoyer für die Sprachkodexforschung. In: PlewnIA, Albrecht / WiTT, Andreas (Hrsg.): Sprachverfall? Dynamik - Wandel - Variation (= Institut für Deutsche Sprache Jahrbuch 2013). Berlin; Boston, S. 219-242.

KLeIN, Wolf Peter (2018): Sprachliche Zweifelsfälle im Deutschen: Theorie, Praxis, Geschichte. Berlin.

NieHAus, Konstantin (2016): Wortstellungsvarianten im Schriftdeutschen: über Kontinuitäten und Diskontinuitäten in neuhochdeutscher Syntax. Heidelberg.

SCHERR, Elisabeth / NiEHAUS, Konstantin (2013): ... weil man den Gebrauchsstandard erheben wird wollen. Variabilität und funktionale Äquivalenz in der Standardsyntax am Beispiel der „Zwischenstellung“ in Verbalkomplexen. In: Hagemann, Jörg / KLein, Wolf Peter / StAFfeldt, Sven (Hrsg.): Pragmatischer Standard. Tübingen, S. 75-84.

WAHRIG (2009): Richtiges Deutsch leicht gemacht. Güterloh.

WAHRIG (2018): Wörterbuch der deutschen Sprache. München. 